\title{
Colloid electrochemistry of conducting polymer: towards potential-induced in-situ drug release
}

Supannee Sankoh, Mikhail Vagin, Alina Sekretareva, Panote Thavarungkul, Proespichaya Kanatharana and Wing Cheung Mak

\section{Journal Article}

\section{Tweet}

N.B.: When citing this work, cite the original article.

Original Publication:

Supannee Sankoh, Mikhail Vagin, Alina Sekretareva, Panote Thavarungkul, Proespichaya Kanatharana and Wing Cheung Mak, Colloid electrochemistry of conducting polymer: towards potential-induced in-situ drug release, Electrochimica Acta, 2017. 228, pp.407-412.

http://dx.doi.org/10.1016/j.electacta.2017.01.028

Copyright: Elsevier

http://www.elsevier.com/

Postprint available at: Linköping University Electronic Press

http://urn.kb.se/resolve?urn=urn:nbn:se:liu:diva-136666

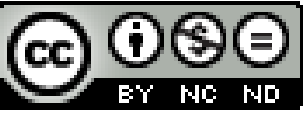




\title{
Colloid electrochemistry of conducting polymer: towards potential- induced in-situ drug release
}

\author{
Supannee Sankoh,\#[a],[b],[c] Mikhail Yu. Vagin, ${ }^{\# *[a],[d]}$ Alina N. Sekretaryova,, ${ }^{[a],[e]}$ \\ Panote Thavarungkul, ${ }^{[b],[f]}$ Proespichaya Kanatharana ${ }^{[b],[c]}$ and Wing Cheung Mak*[a]
}

[a] Department of Physics, Chemistry and Biology, Linköping University, SE-581 83, Linköping, Sweden;

[b] Trace Analysis and Biosensor Research Center, Prince of Songkla University, Hat Yai, Songkla, 90112, Thailand;

[c] Department of Chemistry, Faculty of Science, Prince of Songkla University, Hat Yai, Songkla, 90112, Thailand;

[d] Laboratory of Organic Electronics, Department of Science and Technology, Linköping University, Norrköping, Sweden;

[e] Department of Chemistry, Stanford University, Standford, CA 94305-5080, USA;

[f] Department of Physics, Faculty of Science, Prince of Songkla University, Hat Yai, Songkla, 90112, Thailand.

"These authors contributed equally.

\section{* Corresponding authors:}

Mikhail Yu. Vagin

e-mail: mikva@liu.se

Tel.: +46(0)702753087

Wing Cheung Mak

e-mail:mamak@ifm.liu.se

Tel.: $+46(0) 762674445$ 


\begin{abstract}
:
Over the past decades, controlled drug delivery system remains as one of the most important area in medicine for various diseases. We have developed a new electrochemical control drug release system by combining colloid electrochemistry and electro-responsive microcapsules. The pulsed electrode potential modulation led to the appearance of two processes available for the time-resolved registration in colloid microenvironment: change of the electronic charge of microparticles (from $0.5 \mathrm{~ms}$ to $0.1 \mathrm{~s}$ ) followed by the drug release associated with ionic equilibration (1-10 s). The dynamic electrochemical measurements allow the distinction of drug release associated with ionic relaxation and the change of electronic charge of conducting polymer colloid microparticles. The amount of released drug (methylene blue) could be controlled by modulating the applied potential. Our study demonstrated a surface-potential driven controlled drug release of dispersion of conducting polymer carrier at the electrode interfaces, while the bulk colloids dispersion away from the electrode remains as a reservoir to improve the efficiency of localized drug release. The developed new methodology creates a model platform for the investigations of surface potential-induced in-situ electrochemical drug release mechanism.
\end{abstract}

Keywords: colloid electrochemistry; conducting polymer; potential-modulation; microcapsules; controlled drug release 
Graphical Abstract:

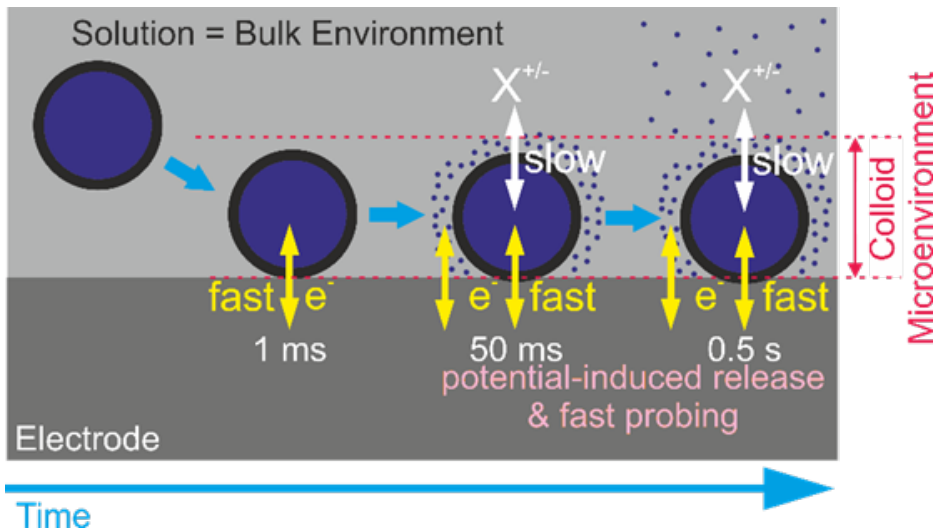

\section{Highlights:}

- Pulsed electrode potential induced an in-situ drug release from dispersion of conducting polymer microcapsules

- Fast detection of the released drug within the colloid microenvironment

- Improved the efficiency of localized drug release at the electrode interface

\section{Introduction}


Over the past decades, controlled drug delivery system remains as one of the most important area in medicine for various applications such as cancer treatments, metabolic disorders, autoimmune diseases and chronic diseases [1-3]. Many different triggering approaches including $\mathrm{pH}$-switch [4], temperatureswitch [5, 6], magnetic field [7], and electrical stimulation [8-12] were utilized for the development of controlled drug release systems. Among all, electrical technique provides a rapid, relatively simple and easy way to control drug release [13].

Conducting polymers such as polypyrrole (PPy) and poly3,4-ethylenedioxythiophene (PEDOT) can be modulated by electrical stimulation to alternate their redox states which results in simultaneous changes in the polymer charges, conductivity and volume allowing to achieve controlled drug release [11, 12, 14]. Electrical stimulation has been utilized to trigger drug release in bulk conducting polymer materials. Due to the combination of biocompatibility $[15,16]$ and the possibility to modulate the properties with electronic charge transfer, PPy attracted an attention for the implantable device engineering. The biodegradability of polypyrrole-based conductive composites might be tailored [17] by means of introduction of degradation sites (e.g. ester linkage) available for in vivo enzymatic cleavage. The products of polymer degradation can be consumed by macrophages during the normal wound healing response [18].

Zinger et. al. first reported on the controlled release system based on PPy film for delivery of glutamate [19]. Thereafter, different dopants have been added to the conducting polymer to develop advanced controlled release systems for anionic, cationic and neutral drugs [13]. Hepel et. al. showed the electrically controlled delivery of chlorpromazine within a melanin-doped PPy film [20, 21]. Bidan et. al. reported using a sulphonated cyclodextrins-doped PPy film for controlled release of N-methylphenothiazine [22]. George et. al. demonstrated the use of a biotin-doped PPy film for electrically controlled delivery of nerve growth factor (NGF)[14]. More recently, nanostructured conducting polymer-based delivery systems have been developed with improved drug delivery efficiency. Luo et. al. reported the use of nano-porous PPy films with increased active surface areas for delivery of fluorescein [23]. The nano-porous PPy film with a higher surface-areas-to-volume ratio exhibited 9 times higher release rate than the bulk planar PPy films. Sharma et. al. developed a macroporous PPy inverse opal film with enhanced loading capacity and release capability of antipsychotic drug risperidone compared to a non-porous PPy film [24]. Beside drug molecules, the biotin-doped porous PPy film has been introduced for electrically controlled release of avidin-functionalized gold nanoparticles [25]. There is a recent reported on the use of a poly(3,4ethylenedioxythiophene)-single walled carbon nanotubes (PEDOT-SWCNTs) composite for electrically controlled delivery of dexamethasone. The SWCNTs greatly improved the conductivity of the film and enhanced the rate of drug release compared with a pure PEDOT film [26]. 
However, the reported electrically controlled drug delivery systems could only perform in bulk polymer films attached to electrode surfaces. In contrast, colloidal drug delivery systems with particulate carriers such as micelles, liposomes, nano- and microparticles have been proven to have excellent ability for drug encapsulation, controlled release and targeting delivery [27]. Different triggering mechanisms based on $\mathrm{pH}[28]$, temperature[29], light[30], enzyme[31], and magnetic-field[32] had been developed for controlled drug release in colloidal delivery systems. Such particle delivery systems have the advantage of small and controllable size, large surface-area-to-volume ratio for maximizing drug loading and enhanced drug release, relatively easy to produce, and more importantly highly dispersible and injectable for site-specific targeted drug delivery[33-35]. The accumulation of drug carrier reaching the targeted tissue could enhance efficiency and effectiveness of the treatment due to the localized delivery of high dosage of the drug close to the targeted tissue surface $[36,37]$. Besides, drug release monitoring in colloidal drug delivery system is challenging, and most detection techniques are mainly measure the released drug in bulk solution [9-11, 14]. However, for targeted drug delivery, it is important to monitor the localized drug release within the microenvironment of the carrier system. By combining the concept of dispersed drug delivery and advance colloid electrochemistry, we have developed the first time an insitu dispersed drug release system based on conducting polymer microcapsules induced by the electrode potential (Figure 1A). The redox conversion of microcapsules colloid solution was accompanied by delayed ionic equilibration yielding the release of methylene blue (MB) as a model ionic drug. The time-resolved discrimination of two processes has been achieved via dynamic electrochemical methods with standard glassy carbon electrode allowing drug release and fast probing of the released drug in the microenvironment. The effect of microscale wall morphology on the conducting polymer capsules and drug release behavior were investigated.

\section{Materials and Methods}

\subsection{Materials}

Calcium chloride $\left(\mathrm{CaCl}_{2}\right)$, copper (II) perchlorate $\left(\mathrm{Cu}\left(\mathrm{ClO}_{4}\right)_{2} \bullet 6 \mathrm{H}_{2} \mathrm{O}\right)$, sodium carbonate $\left(\mathrm{Na}_{2} \mathrm{CO}_{3}\right)$, ethylenediaminetetracetic acid (EDTA), ethanol, 2-propanol, methylene blue (MB) and pyrrole (99\%) were purchased from Sigma-Aldrich and were used as received. Pyrrole (99\%) was further purified before use by passing through a neutral column of alumina $(0.05 \mathrm{~nm})$ to obtain a colorless liquid. Water was purified using a Milli-Q water purification system.

\subsection{Fabrication of the MB-loaded PPy microparticle drug carrier}


PPy microparticles (PPyMP) were prepared by hard template polymerization as reported previously [38]. In brief, $1 \mathrm{~mL}$ of $\mathrm{CaCl}_{2}(0.5 \mathrm{M})$ and $1 \mathrm{~mL}$ of $\mathrm{Na}_{2} \mathrm{CO}_{3}(0.5 \mathrm{M})$ were rapidly mixed at 600 r.p.m. and kept under stirring for 30 seconds to produce calcium carbonate $\left(\mathrm{CaCO}_{3}\right)$ microparticles as a template. $\mathrm{CaCO}_{3}$ microparticles were washed twice with water consecutively, followed by washing with ethanol and 2propanol, by centrifugation (2000 rpm, $1 \mathrm{~min}$ ) and re-dispersion cycles. $200 \mu \mathrm{L}$ of MB solution $(50 \mathrm{mM}$ ) in pure pyrrole monomer were mixed with $\mathrm{CaCO}_{3}$ templates followed by incubation for 60 minutes at room temperature, allowing the $\mathrm{MB}$ and monomer loading. The pyrrole-MB-loaded $\mathrm{CaCO}_{3}$ microparticles were centrifuged (2000 r.p.m., $1 \mathrm{~min}$ ) and the supernatant was discarded to remove the non-absorbed MB and monomers. A chemical polymerization of MB-loaded PPyMP was carried out by mixing the resulting pyrrole-MB-loaded $\mathrm{CaCO}_{3}$ microparticles with $1 \mathrm{~mL}$ of $\mathrm{Cu}\left(\mathrm{ClO}_{4}\right)_{2} \cdot 6 \mathrm{H}_{2} \mathrm{O}$ in propanol and allow polymerization for 1,3 and 6 hours under shaking. $\mathrm{CaCO}_{3}$ microparticles containing the synthesized MBloaded PPyMP were washed consecutively twice following the sequence of 2-propanol, ethanol and MilliQ water by centrifugation (2000 r.p.m., $1 \mathrm{~min}$ ) and re-dispersion cycles. Subsequently, $\mathrm{CaCO}_{3}$ template was removed by the addition of $1 \mathrm{~mL}$ EDTA solution ( $0.2 \mathrm{M})$ followed by an incubation for 1 hour at room temperature. Finally, the acquired MB-loaded PPyMP were washed twice with Milli-Q water by centrifugation (2000 rpm, $1 \mathrm{~min}$ ) and re-dispersion cycles.

\subsection{Particle size and zeta potentials analysis}

The particle size and zeta potential (surface charge) of the microparticles in solution were measured using a Zetasizer Nano ZS90 (Malvern Instruments Ltd., Worcestershire, UK). The particle size was measured based on a dynamic light scattering technique, measuring the Brownian motion of the particles and converting the data into a size distribution graph using the Stokes-Einstein relationship. The zeta potential measurement is based on laser Doppler micro-electrophoresis principle. The electrophoretic mobility $(\mu)$

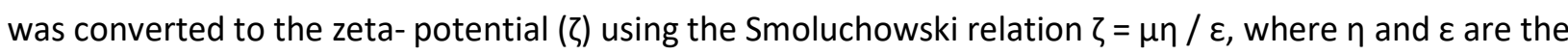
viscosity and permittivity of the solution respectively. $1 \mathrm{~mL}$ of suspended microparticles in PBS solution was loaded into a zeta potential measuring cell. The measurements were performed at 25 o $\mathrm{C}$ and the mean zeta potential values were calculated by taking an average of 3 repeated measurements.

\subsection{Electrochemical measurements}

All electrochemical experiments were performed with a PGSTAT30 potentiostat (Autolab, Netherlands) under NOVA or GPES software control employing a conventional three-electrode electrochemical cell. All experiments were performed in aqueous media (10 mM PBS pH 7.4). The oxygen background effect was eliminated by bubbling nitrogen for 15 minute. All the measurements have been carried out under 
nitrogen blanket. The glassy carbon electrode (GCE, $3 \mathrm{~mm}$ diameter with a surface area (electrode footprint) of $0.0707 \mathrm{~cm} 2$ was utilized for the current densities calculations) was used as the working electrode, a platinum wire as the auxiliary electrode, and a silver/silver chloride electrode as the reference electrode $(3 \mathrm{M} \mathrm{KCl})$. Prior to use, the working electrode was successively polished with 1.0, 0.3 and 0.05 $\mu \mathrm{m}$ alumina powders and sonicated in water for $10 \mathrm{~min}$ after each polishing step. Finally, the electrode was washed with ethanol and then dried with a high purity argon stream. The GCE was modified with PPyMP by drop-casting of aqueous solutions followed by curing at 60 ㄷ $\mathrm{C}$ for $30 \mathrm{~min}$. Potential modulation of MB-loaded PPyMP were performed by using GCE modified with drop-casting of MB-loaded PPyMP or measuring the MB-loaded PPyMP stable colloidal solution.

\section{Results and Discussion}

\subsection{Characterizations of MB-loaded PPyMP microparticle carrier}

MB-loaded polypyrrole microparticles (PPyMP) with tunable wall thickness were fabricated with $\mathrm{CaCO}_{3}$ template-assisted time dependent polymerization technique. By controlling the polymerization time ranging from 1, 3 and 6 hour(s), we were able to obtain MB-loaded PPyMP with different wall thickness (Figure 1B-D). We can observe that the MB-loaded PPyMP fabricated with shorter polymerization time appeared capsule-like morphology with a slightly transparent center region, while the MB-loaded PPyMP fabricated with longer polymerization time appeared darker in color resulting from the increase of the wall thickness. All the synthesized MB-loaded PPyMPs with different polymerization times of 1, 3 and 6 hour(s) were fairly homogenous with average diameters of $2.32 \pm 0.61,2.14 \pm 0.63$ and $2.44 \pm 0.47 \mu \mathrm{m}$, respectively. The narrow size distribution of the MB-loaded PPyMPs results from the advantage of $\mathrm{CaCO}_{3}$ templated fabrication method. The morphologies of the MB-loaded PPyMPs were further examined with a high resolution SEM (Figure 1E-G). The morphology of the MB-loaded PPyMPs fabricated with shorter polymerization times ( 1 and 3 hours) appeared flatten with folds and creases structure resulted from the collapsed hollow microparticles due to high vacuum condition during SEM imaging, while the MB-loaded PPyMP fabricated with 6 hours polymerization shows a more intact non-flatten morphology. The thickness of the 1, 3 and 6 hour(s) PPyMPs was estimated to be 100,205 and $310 \mathrm{~nm}$, respectively, by measuring thickness of the fold structure (which is equivalent to twice of the wall thickness of the dried collapsed MB-loaded PPyMP). Zeta potential measurements were used to characterize the surface charge of the MB-loaded PPyMP. MB-loaded PPyMP prepared with different polymerization times of 1, 3 and 6 hour(s) had average positive zeta potential values of $28.8 \pm 5.9,27.2 \pm 8.3$ and $30.8 \pm 5.5 \mathrm{mV}$, respectively. The positive charge results from the protonated nitrogen atom of polypyrrole at $\mathrm{pH} 7.4$ [34] and from monocationic form of $\mathrm{MB}$ (pKa 5.6). 


\subsection{Electrochemical characterizations}

The effect of the structural organization on the material characteristics of drug storage and release was investigated first by cyclic voltammetry at glassy carbon electrode (GCE) modified with the same loading mass (50 $\mu \mathrm{g}$ calculated from the dry weight) of the MB-loaded PPyMPs with different morphologies, while having similar chemical composition, surface zeta potential and physical diameter. A change in the electronic charge due to the oxidation and reduction processes of the conducting polymers is accompanied by an equivalent change in the ionic charge for the electroneutrality maintaining, which requires ionic mass transport between the polymer and electrolyte $[10,39]$ yielding the volume change [40]. The magnitude of expansion and contraction depends on the number and size of transferred ions [41] opening wide possibilities for the applications [42], e.g. for controllable drug release [1] and microfluidic pumps [43].

The MB storage capacity of developed materials has been studied by cyclic voltammetry with electrodes modified with drop-casted films fabricated from thin-, medium and thick-hollow MB-loaded PPyMPs (Figure. 2). On the contrast to buffer, the presence of MB-loaded PPyMPs in the solution led to the appearance of $\mathrm{MB}$ reversible redox process at $-0.3 \mathrm{~V}$ and slightly larger capacitive currents. Moreover, the solutions became blue after the measurements. These facts illustrate the presence of the MB inside the PPyMPs available for the release by the applied potential during voltammetry experiment. The scan rate study (Figure. 1S) showed a surface-confined behavior of observed MB redox process due to the adsorption of released monocation drug onto GCE surface [44].

In order to decouple the contributions from PPy and MB into the voltammetric response of the filmmodified electrodes the measurements were carried out in acidic media ensuring higher PPy conductivity (Figure 2S). The well-defined couple of peaks associated with MB redox reaction is visible over the boxshaped electrocapacitive voltammogram of PPy allowing the estimation of the background-subtracted charges under the $\mathrm{MB}$ redox peaks obtained at low scan rate $(5 \mathrm{mV} / \mathrm{s})$. The MB-doped thin-, medium- and thick-wall hollow PPyMP films showed the values of $0.19 \mathrm{mC}, 0.14 \mathrm{mC}$ and $0.1 \mathrm{mC}$, respectively. Assuming two electron $\mathrm{MB}$ redox process and knowing the masses of films, the values of $\mathrm{MB}$ content were estimated as $0.66 \%, 0.53 \%$ and $0.40 \%$ for thin-, medium- and thick-wall hollow PPyMP. Having the largest inner cavity, the thin-wall hollow microparticles synthesized during short reaction time (1 hour) showed the largest MB content. Therefore, the main storage domain of the host material is the inner cavity of the hollow microparticle. MB remains intact and available for the release from PPy polymerized in mild conditions.

\subsection{Potential-induced drug released and fast probing of released drug}


In order to get the insight into the mechanism of the observed potential-induced drug release the dynamic electrochemical methods, i.e. double step chronocoulometry and fast voltammetry, have been utilized for the studies of MB-loaded PPyMP colloid solutions. In order to prevent the passive leakage of MB from the carrier microparticles due to concentration gradient the concentrated colloid solutions have been washed by centrifugation with PBS buffer just before the addition into the electrochemical cell. No turbidity was observed after the introduction of MB-loaded PPyMP into the measurement cell illustrating the stability of colloid solution.

First modulation pulse of the double-step chronocoulometry was utilized in order to affect the colloid solution of microparticles, which might lead to the drug release due to the appearance of ionic flow to equilibrate the change in the electronic charge of the particles. The modulation pulse of applied potential should trigger the complex phenomena in colloid solution appeared in a close vicinity to the working electrode surface. Firstly, after the fast relaxation of double layer on electrode surface the applied potentials should lead to the electronic charge change of the conducting polymer microparticles. Secondly, the ionic charge should appear as a response to the first process in order to maintain electroneutrality of the material resulting in the possible drug release. Both electronic and ionic current flows should be timeresolved due to the general difference in the rates. The second pulse of the double-step chronocoulometry was designed to detect the changes in the microenvironment caused by the modulation pulse.

Figure $3 \mathrm{~A}$ represents the background-subtracted integral charge transients obtained in the colloid solutions of pristine and MB-loaded PPyMP after the same-length modulation pulses of two opposite amplitudes: $-0.7 \mathrm{~V}$ and $0.7 \mathrm{~V}$. As a background the integral charge transients have been obtained in pure background electrolytes. It is seen, that both materials caused the appearance of the visible anodic and cathodic charge injections into the bulk of the colloid solution illustrating the possibilities of charge injection in the bulk of electrolyte. The polarization of the modulation pulse defines the direction of detected current flow. The presence of MB significantly increase anodic and cathodic charge injection efficiencies, which might be a result of two effects related to electronic and ionic processes: increase of electronic conductivity of MB-loaded PPyMP [45] and the presence of MB undergoing subsequent faradaic reactions on the working electrode surface:

$\mathrm{MB}^{+}+\mathrm{H}^{+}+2 \mathrm{e}^{-}=\mathrm{LMB}$

where $L M B$ is a fully reduced leuco-MB. The possible monoelectronic reduction caused the appearance of a variety of intermediates stable on the electrode surface [44].

In order to distinguish electronic and ionic processes the modulation time dependence has been studied (Figure. 3B). The increase of charge with increase of modulation time is shown for all measurements illustrating the charge transfer kinetics of modulation detectable at the second pulse. Higher amplitudes 
of modulation potential led to the larger detectable charges showing increase of charge injection efficiency with polarization increase. The short times of the modulation (from $0.5 \mathrm{~ms}$ to $0.1 \mathrm{~s}$ ) are characterized with the increase of detectable charges followed by the saturation at longer times of modulation pulse (1-10 s). The saturation threshold observed for all modulation pulses probably illustrate the appearance of the diffusion of the converted forms of colloid material from the electrode surface. Importantly, the charge detected at the long modulation pulse of large anodic amplitude ( $0.7 \mathrm{~V})$ revealed the prominent dependence on the potential of the detection pulse (Inset in Figure 3B). The pristine PPyMP showed a minor increase of additional charge (Figure. 3S). The increase of cathodic polarization of the detection pulse led to the larger detectable charges (23\%), which illustrates the additional faradaic contribution featured with slower kinetics in comparison with the general redox process of polymer microparticles appeared at shorter times and characterized with the independence on the detection potential. The slow rate of additional faradaic process observed at modulation pulse of large anodic amplitude demonstrates the involvement of slow ionic process, which controls the release of the drug contributing to the detectable charge. The rest of the modulation pulse amplitudes showed minor dependencies on the polarization of the detection pulse (i.e. $17 \%$ for $0.3 \mathrm{~V}$ and for $-0.1 \mathrm{~V}, 9 \%$ for $-0.7 \mathrm{~V}$ ). Therefore, the efficiency of the faradaic contribution from the redox conversion of MB depends on the modulation pulse amplitude. The largest contribution from of the redox process of released drug was observed at the modulation of $0.7 \mathrm{~V}$ (Figure 4) associated with the release of monocation drug available for detection via mono- and two-electronic reduction [44]. The clear transition between time-dependent and independent modes of faradaic process of released MB observed at $0.5 \mathrm{~s}$ might be rationalized by the diffusion layer thickness, assuming the planar diffusion on the released $M B$ at the electrode surface. Diffusion layer thickness estimated as: $\delta=\sqrt{\pi D t}$, where $t$ is the transition time $(\mathrm{s})$ and $D$ is the MB diffusion coefficient $\left(0.6 \times 10^{-9}-0.8 \times 10^{-9}\left(\mathrm{~m}^{2} \mathrm{~s}^{-1}\right)[46]\right.$ was 30-35 $\mu \mathrm{m}$, which is larger than the average PPyMP diameter.

In order to confirm the effect by independent measurements the modulation pulse has been followed by fast voltammetry measurements as a detection step (Figure 5). The comparison of voltammograms obtained in the colloid solution of MB-loaded PPyMP before and after modulation pulse of high anodic amplitude $(0.7 \mathrm{~V})$ showed the appearance of redox peak currents of $M B$ illustrating the presence of $M B$ released into the buffer. The fast voltammetry allowed the visualization of the released drug, which diffused away due to the concentration gradient disabling the detection with slower scan rates. Importantly, the thin-wall hollow MB-doped showed the largest redox currents of the drug, while the medium-wall hollow microparticles showed significantly smaller peaks. The thick-wall hollow microparticles showed the absence of the MB redox signal. Therefore, in consistency with drop-casted films the measurements performed with a colloid solution confirmed the microscale morphology control of the amount of loaded drug, which is available of potential-induced release. 


\section{Conclusion}

In conclusion, we demonstrated the feasibility for precision control of drug release from dispersed electroresponsive microcapsules triggered by in-situ colloids electrochemistry occurring at the electrode surface, while the bulk colloids dispersion away from the electrode surface remains as a drug reservoir, thus improving the efficiency of localized drug release at the target location. The dynamic electrochemical measurements allow the distinction of drug release associated with ionic relaxation and the change of electronic charge of conducting polymer microparticles. The developed new methodology creates a model platform for the investigations of surface potential-induced in-situ release mechanism with a long term vision on development of a direct controlled drug release system for biological objects driven by cell surface potentials.

\section{Acknowledgements}

Supports for Supannee Sankoh from the Royal Golden Jubilee Ph.D-program (RGJ) supported by The Thailand Research Fund (3.C.PS/51/B.1) is gratefully acknowledged.

\section{References}

[1] M.R. Abidian, D.H. Kim, D.C. Martin, Conducting-polymer nanotubes for controlled drug release, Advanced Materials, 18 (2006) 405-+.

[2] Y.Q. Huo, S.B. Zhang, H.Y. Zhang, Capacitive Performance of Polyaniline/Manganese Dioxide Nanofiber Microsphere, Journal of Applied Polymer Science, 131 (2014) 40575.

[3] L. Qie, W. Chen, H. Xu, X. Xiong, Y. Jiang, F. Zou, X. Hu, Y. Xin, Z. Zhang, Y. Huang, Synthesis of functionalized 3D hierarchical porous carbon for high-performance supercapacitors, Energy \& Environmental Science, 6 (2013) 2497-2504.

[4] P. Gupta, K. Vermani, S. Garg, Hydrogels: from controlled release to $\mathrm{pH}$-responsive drug delivery, Drug Discovery Today, 7 (2002) 569-579.

[5] J.E. Chung, M. Yokoyama, T. Okano, Inner core segment design for drug delivery control of thermoresponsive polymeric micelles, Journal of Controlled Release, 65 (2000) 93-103.

[6] N. Yamada, T. Okano, H. Sakai, F. Karikusa, Y. Sawasaki, Y. Sakurai, Thermoresponsive polymeric surfaces - control of attachment and detachment of cultured cells, Makromolekulare Chemie-Rapid Communications, 11 (1990) 571-576. 
[7] D. Pan, H. Zhang, T. Fan, J. Chen, X. Duan, Nearly monodispersed core-shell structural Fe304@DFURLDH submicro particles for magnetically controlled drug delivery and release, Chemical Communications, 47 (2011) 908-910.

[8] M.Y. Bai, Y.J. Cheng, S.A. Wickline, Y.N. Xia, Colloidal Hollow Spheres of Conducting Polymers with Smooth Surface and Uniform, Controllable Sizes, Small, 5 (2009) 1747-1752.

[9] J.M. Pernaut, J.R. Reynolds, Use of conducting electroactive polymers for drug delivery and sensing of bioactive molecules. A redox chemistry approach, Journal of Physical Chemistry B, 104 (2000) 4080-4090. [10] S. Sirivisoot, R. Pareta, T.J. Webster, Electrically controlled drug release from nanostructured polypyrrole coated on titanium, Nanotechnology, 22 (2011).

[11] Y. Takeoka, T. Aoki, K. Sanui, N. Ogata, M. Yokoyama, T. Okano, Y. Sakurai, M. Watanabe, Electrochemical control of drug-release from redox-active micelles, Journal of Controlled Release, 33 (1995) 79-87.

[12] R. Wadhwa, C.F. Lagenaur, X.T. Cui, Electrochemically controlled release of dexamethasone from conducting polymer polypyrrole coated electrode, Journal of Controlled Release, 110 (2006) 531-541.

[13] D. Svirskis, J. Travas-Sejdic, A. Rodgers, S. Garg, Electrochemically controlled drug delivery based on intrinsically conducting polymers, Journal of Controlled Release, 146 (2010) 6-15.

[14] P.M. George, D.A. LaVan, J.A. Burdick, C.Y. Chen, E. Liang, R. Langer, Electrically controlled drug delivery from biotin-doped conductive polypyrrole, Advanced Materials, 18 (2006) 577-+.

[15] C.E. Schmidt, V.R. Shastri, J.P. Vacanti, R. Langer, Stimulation of neurite outgrowth using an electrically conducting polymer, Proceedings of the National Academy of Sciences of the United States of America, 94 (1997) 8948-8953.

[16] X.Y. Cui, V.A. Lee, Y. Raphael, J.A. Wiler, J.F. Hetke, D.J. Anderson, D.C. Martin, Surface modification of neural recording electrodes with conducting polymer/biomolecule blends, Journal of Biomedical Materials Research, 56 (2001) 261-272.

[17] T.J. Rivers, T.W. Hudson, C.E. Schmidt, Synthesis of a novel, biodegradable electrically conducting polymer for biomedical applications, Advanced Functional Materials, 12 (2002) 33-37.

[18] T.R. Green, J. Fisher, J.B. Matthews, M.H. Stone, E. Ingham, Effect of size and dose on bone resorption activity of macrophages by in vitro clinically relevant ultra high molecular weight polyethylene particles, Journal of Biomedical Materials Research, 53 (2000) 490-497.

[19] B. Zinger, L.L. Miller, Timed release of chemicals from polypyrrole films, Journal of the American Chemical Society, 106 (1984) 6861-6863.

[20] M. Hepel, J. Fijalek, Electrorelease of Drugs from Composite Polymer Films, in: R. Ottenbrite (Ed.) Polymeric Drugs and Drug Administration, ACS Symposium Series, Washington D.C., 1994, pp. 79-97.

[21] M. Hepel, F. Mahdavi, Application of the electrochemical quartz crystal microbalance for electrochemically controlled binding and release of chlorpromazine from conductive polymer matrix, Microchemical Journal, 56 (1997) 54-64. 
[22] G. Bidan, C. Lopez, F. Mendesviegas, E. Vieil, A. Gadelle, Incorporation of sulfonated cyclodextrins into polypyrrole - an approach for the electro-controlled delivering of neutral-drugs, Biosensors \& Bioelectronics, 10 (1995) 219-229.

[23] X.L. Luo, X.T. Cui, Electrochemically controlled release based on nanoporous conducting polymers, Electrochemistry Communications, 11 (2009) 402-404.

[24] M. Sharma, G.I.N. Waterhouse, S.W.C. Loader, S. Garg, D. Svirskis, High surface area polypyrrole scaffolds for tunable drug delivery, International Journal of Pharmaceutics, 443 (2013) 163-168.

[25] B.G. Choi, J. Hong, W.H. Hong, P.T. Hammond, H. Park, Facilitated Ion Transfer in All-Solid-State Flexible Supercapacitors, ACS Nano, 5 (2011) 7205-7213.

[26] Y.H. Xiao, X.X. Ye, L. He, J.F. Che, New carbon nanotube-conducting polymer composite electrodes for drug delivery applications, Polymer International, 61 (2012) 190-196.

[27] L. Yang, P. Alexandridis, Physicochemical aspects of drug delivery and release from polymer-based colloids, Current Opinion in Colloid \& Interface Science, 5 (2000) 132-143.

[28] E.R. Gillies, T.B. Jonsson, J.M.J. Frechet, Stimuli-responsive supramolecular assemblies of lineardendritic copolymers, Journal of the American Chemical Society, 126 (2004) 11936-11943.

[29] C.H. Chang, P.S. Son, J.-A. Yoon, S.-H. Choi, Synthesis of Hollow Conductive Polypyrrole Balls by the Functionalized Polystyrene as Template, Journal of Nanomaterials, Article ID 168025 (2010) 1-6.

[30] J. Lademann, H. Richter, F. Knorr, A. Patzelt, M.E. Darvin, E. Ruhl, K.Y. Cheung, K.K. Lai, R. Renneberg, W.C. Mak, Triggered release of model drug from AuNP-doped BSA nanocarriers in hair follicles using IRA radiation, Acta Biomaterialia, 30 (2016) 388-396.

[31] W.C. Mak, A. Patzelt, H. Richter, R. Renneberg, K.K. Lai, E. Ruhl, W. Sterry, J. Lademann, Triggering of drug release of particles in hair follicles, Journal of Controlled Release, 160 (2012) 509-514.

[32] P. Dames, B. Gleich, A. Flemmer, K. Hajek, N. Seidl, F. Wiekhorst, D. Eberbeck, I. Bittmann, C. Bergemann, T. Weyh, L. Trahms, J. Rosenecker, C. Rudolph, Targeted delivery of magnetic aerosol droplets to the lung, Nature Nanotechnology, 2 (2007) 495-499.

[33] J.A. Champion, Y.K. Katare, S. Mitragotri, Particle shape: A new design parameter for micro- and nanoscale drug delivery carriers, Journal of Controlled Release, 121 (2007) 3-9.

[34] A. Kumari, S.K. Yadav, S.C. Yadav, Biodegradable polymeric nanoparticles based drug delivery systems, Colloids and Surfaces B-Biointerfaces, 75 (2010) 1-18.

[35] W.C. Mak, H. Richter, A. Patzelt, W. Sterry, K.K. Lai, R. Renneberg, J. Lademann, Drug delivery into the skin by degradable particles, European Journal of Pharmaceutics and Biopharmaceutics, 79 (2011) 23-27. [36] U.K. Marelli, F. Rechenmacher, T.R.A. Sobahi, C. Mas-Moruno, H. Kessler, Tumor Targeting via Integrin Ligands, Frontiers in oncology, 3 (2013) 222-222.

[37] O. Tredan, C.M. Galmarini, K. Patel, I.F. Tannock, Drug resistance and the solid tumor microenvironment, Journal of the National Cancer Institute, 99 (2007) 1441-1454. 
[38] R. Wannapob, M.Y. Vagin, I. Jeerapan, W.C. Mak, Pure nanoscale morphology effect enhancing energy storage characteristics of processable hierarchical polypyrrole., Langmuir, 31 (2015) 11904-11913. [39] E. Smela, Conjugated polymer actuators for biomedical applications, Advanced Materials, 15 (2003) 481-494.

[40] E. Smela, O. Inganas, I. Lundstrom, Controlled folding of micrometer-size structures, Science, 268 (1995) 1735-1738.

[41] M.R. Gandhi, P. Murray, G.M. Spinks, G.G. Wallace, Mechanism of electrochemical actuation in polypyrrole, Synthetic Metals, 73 (1995) 247-256.

[42] Q.B. Pei, O. Inganas, Conjugated polymers and the bending cantiliver method - electrical muscles and smart devices, Advanced Materials, 4 (1992) 277-278.

[43] S.B. Adeloju, S.J. Shaw, G.G. Wallace, Pulsed-amperometric detection of urea in blood samples on a conducting polypyrrole-urease biosensor, Analytica Chimica Acta, 341 (1997) 155-160.

[44] M. Hepel, W. Janusz, Study of leuco-methylene blue film growth and its reoxidation on sulphurmodified Au-EQCN electrode, Electrochimica Acta, 45 (2000) 3785-3799.

[45] M.Y. Vagin, I. Jeerapan, R. Wannapob, P. Thavarungkul, P. Kanatharana, N. Anwar, T. McCormac, M. Eriksson, A.P.F. Turner, E.W.H. Jager, W.C. Mak, Water-processable polypyrrole microparticle modules for direct fabrication of hierarchical structured electrochemical interfaces., Electrochimica Acta, 190 (2016) 495-503.

[46] D.G. Leaist., The effect of aggregation, counterion binding, and added $\mathrm{NaCl}$ on diffusion of aqueous methylene blue, Can. J. Chem., 66 (1988) 2452-2457. 


\section{Figure Captions}

Figure 1. A) Scheme of potential-induced time-resolved controlled drug release of the dispersed microcapsules, followed by simultaneous detection of the released drug within the colloid microenvironment. (B - D) Phase contrast images and (E-G) SEM images of MB-loaded PPyMPs fabricated with polymerization time of 1,3 and 6 hours, respectively.

Figure 2. The microscale morphology control of the storage capacity of MB-loaded PPyMP. The cyclic voltammograms were recorded before and after GCE electrode modification with drop-casted films formed from the thin-, medium- and thick-wall MB-loaded PPyMP (red, blue and magenta curves, respectively; PBS buffer, scan rate $50 \mathrm{mV} / \mathrm{s}$, mass of the material $50 \mu \mathrm{g}$ ).

Figure 3. Potential-induced drug release investigated by double-step chronocolulometry. A) The dependencies of background-subtracted charges on the elapsed time of detection recorded in colloid solutions of MB-loaded and pristine PPyMP after modulation pulse of $0.05 \mathrm{~s}$. B) The dependencies of background-subtracted charges on the time of modulation pulses $(-0.7 \mathrm{~V},-0.1 \mathrm{~V}, 0.3 \mathrm{~V}$ and $0.7 \mathrm{~V}$ as black, red, blue and green symbols, respectively) measured at different detection potentials $(0.5 \mathrm{~s},-0.1 \mathrm{~V}$ and $0.4 \mathrm{~V}$ as open and filled symbols, respectively). Inset: the dependence of the background-subtracted charge detected after $1 \mathrm{~s}$ of modulation pulse $(0.7 \mathrm{~V})$ on the detection potential $(0.5 \mathrm{~s})$.

Figure 4. The dependencies of differences between background-subtracted charges measured at $-0.1 \mathrm{~V}$ and $-0.4 \mathrm{~V}(0.5 \mathrm{~s}$ ) on the time of modulation pulses (amplitudes of $-0.7 \mathrm{~V},-0.1 \mathrm{~V}, 0.3 \mathrm{~V}$ and $0.7 \mathrm{~V}$ as black ( $\nabla$ and $\square$ - MB-doped and pristine PPyMP, respectively), red $(\mathbf{\Delta})$, blue (0) and green ( $\boldsymbol{\nabla})$ symbols, respectively).

Figure 5. The detection of potential-induced drug release with fast voltammetry. The fast cyclic voltammograms $(5 \mathrm{~V} / \mathrm{s})$ were recorded at GCE in pure PBS (black curves) and in colloid solution of thin$(A)$, medium- $(B)$ and thick-hollow $(C)$ MB-loaded PPyMP before and after modulation potential pulse (red and blue curves, respectively; $0.05 \mathrm{~s}, 0.7 \mathrm{~V})$. 


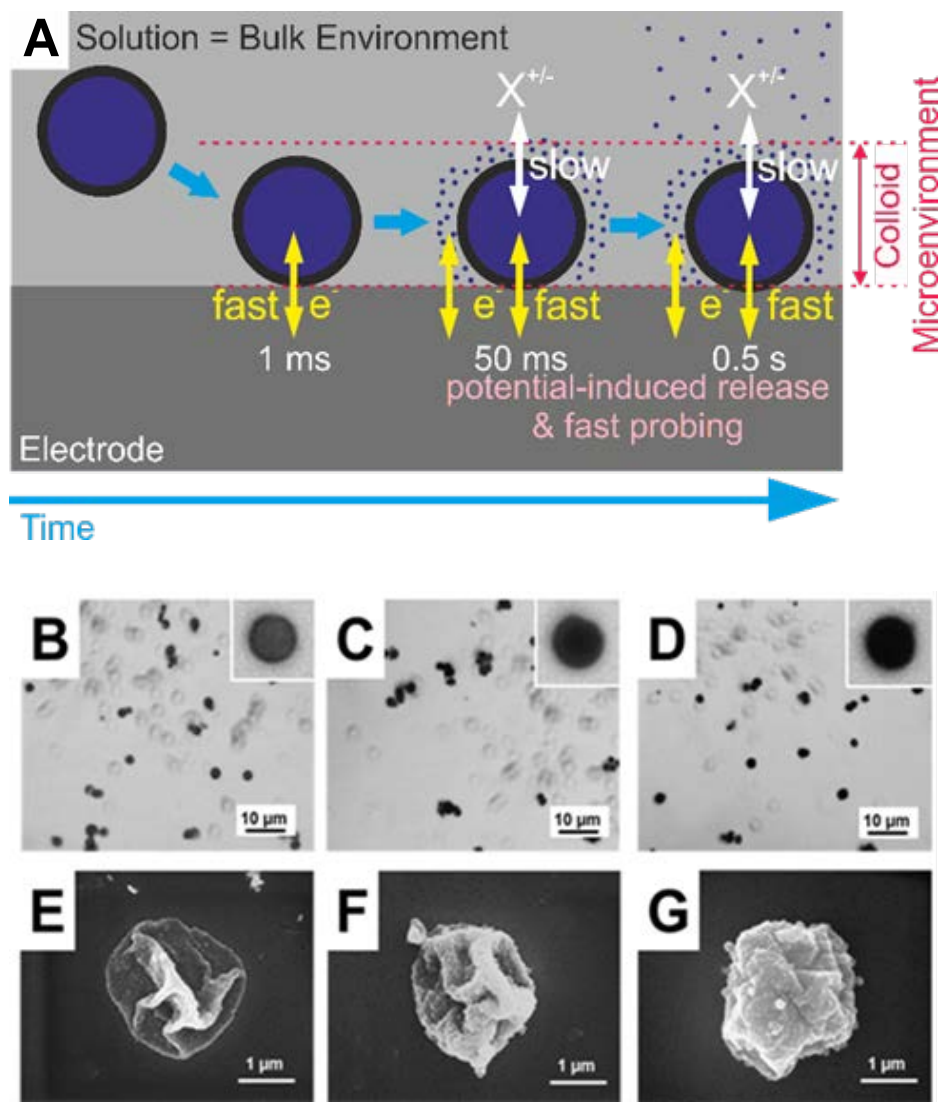

Figure 1 


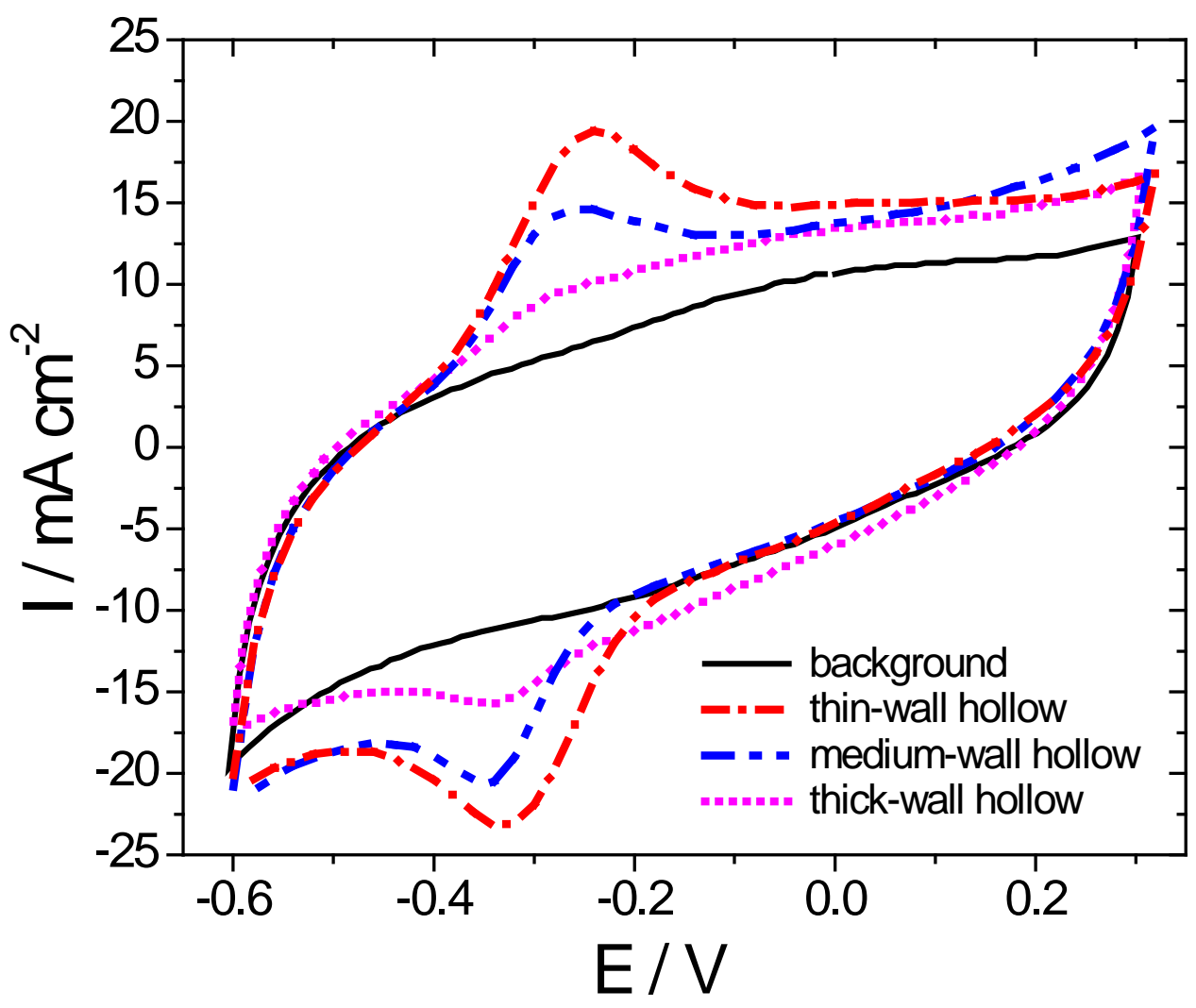

Figure 2 


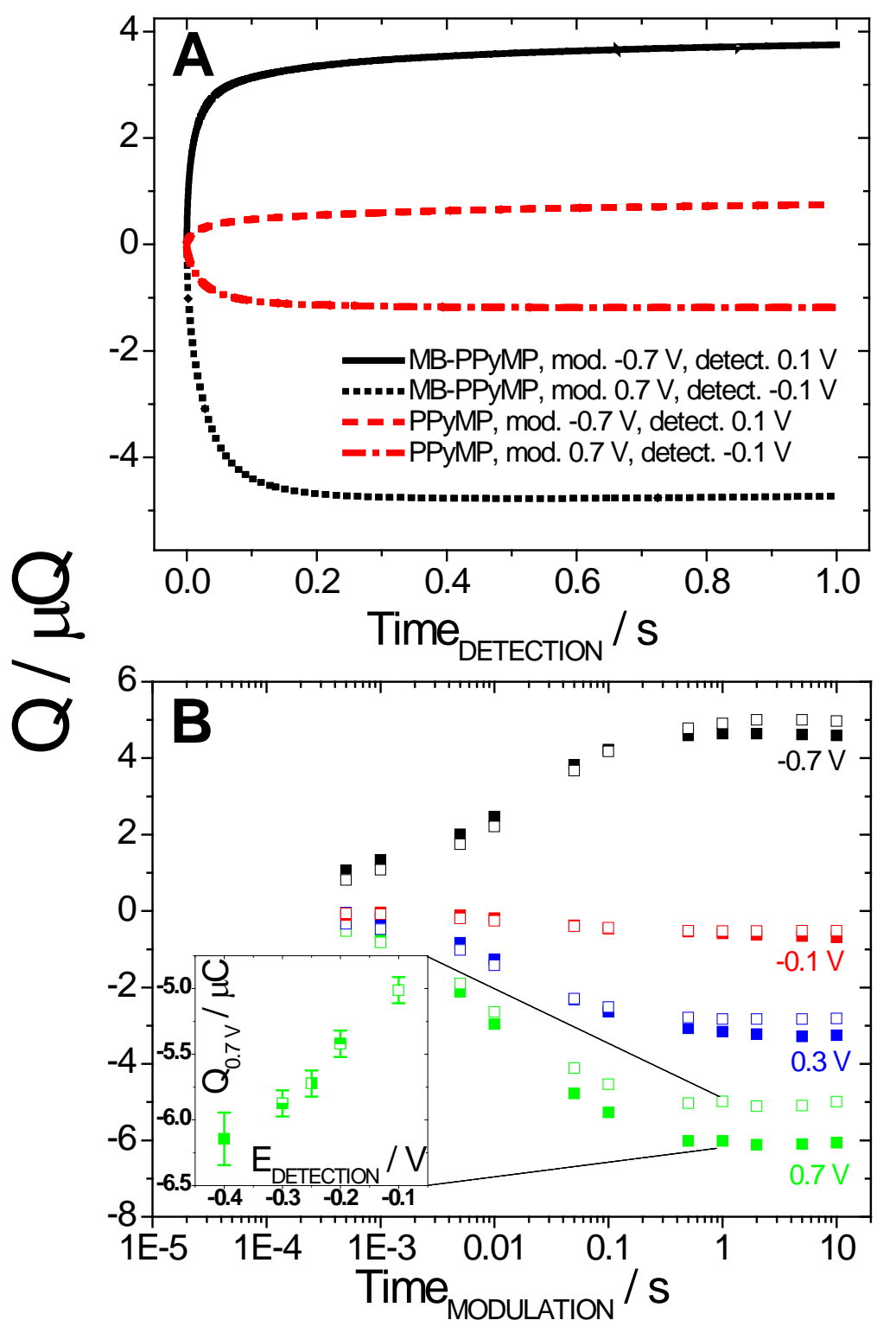

Figure 3 


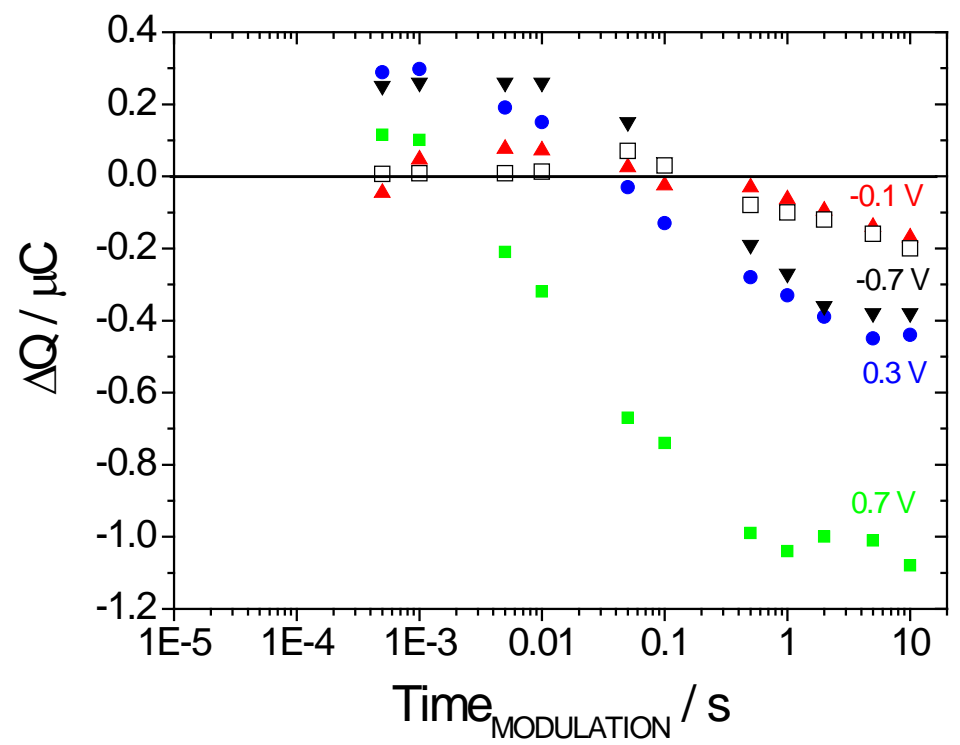

Figure 4 


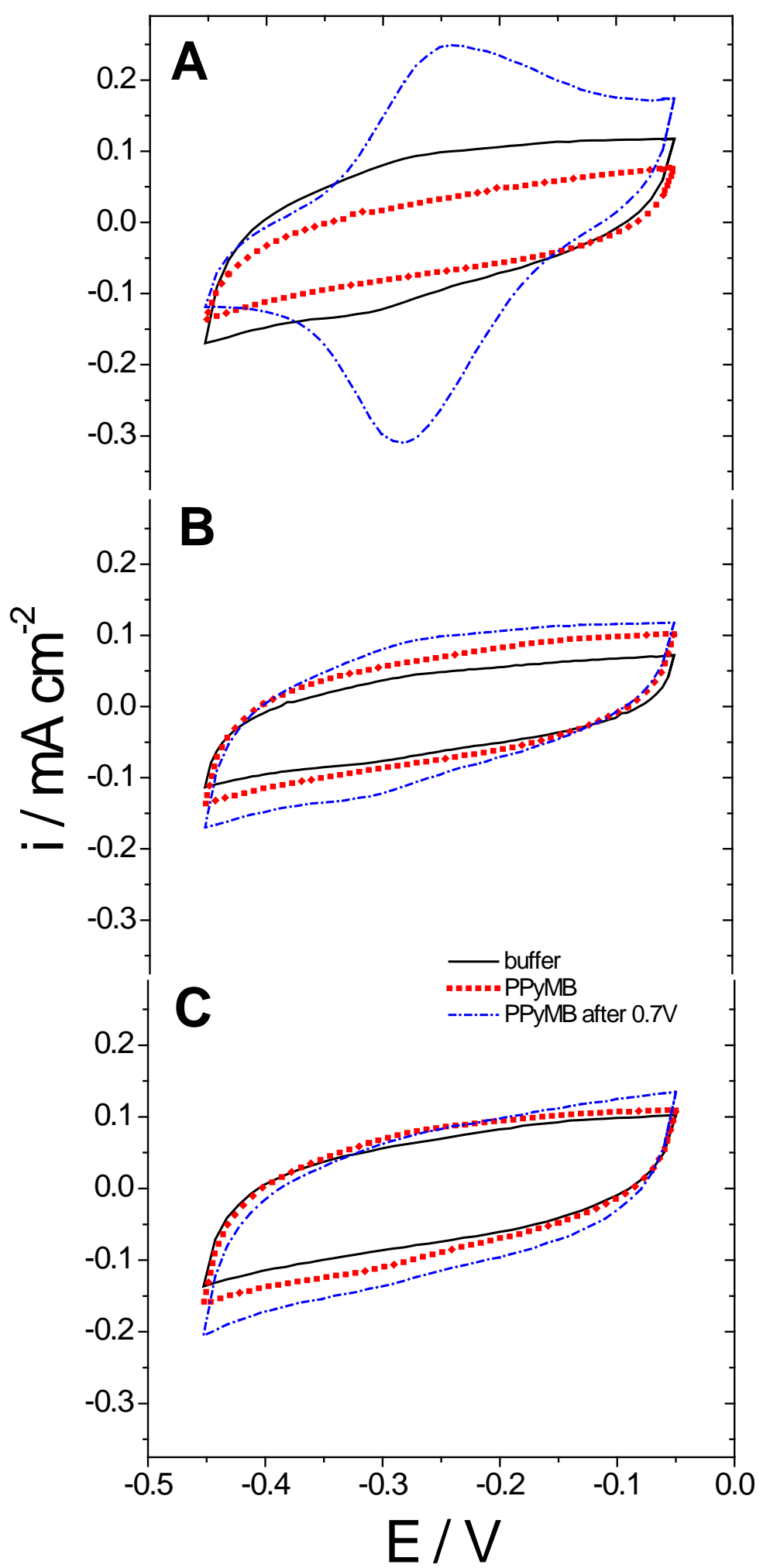

Figure 5 